\title{
Représenter une reine de France. Marie de Médicis et le cycle de Rubens au palais du Luxembourg
}

\section{Fanny Cosandey}

\section{(2) OpenEdition \\ 1 Journals}

Édition électronique

URL : http://journals.openedition.org/clio/645

DOI : 10.4000/clio.645

ISSN : 1777-5299

Éditeur

Belin

Édition imprimée

Date de publication : 1 avril 2004

ISSN : 1252-7017

Référence électronique

Fanny Cosandey, «Représenter une reine de France. Marie de Médicis et le cycle de Rubens au palais du Luxembourg ", Clio. Femmes, Genre, Histoire [En ligne], 19 | 2004, mis en ligne le 27 novembre 2006, consulté le 01 mai 2019. URL : http://journals.openedition.org/clio/645 ; DOI : 10.4000/clio.645

Ce document a été généré automatiquement le 1 mai 2019.

Tous droits réservés 


\title{
Représenter une reine de France. Marie de Médicis et le cycle de Rubens au palais du Luxembourg
}

\author{
Fanny Cosandey
}

1 Unique exemple d'un cycle commandé par une reine de France pour mettre en scène sa propre histoire, la série de tableaux réalisés par Rubens entre 1622 et 1625 offre un témoignage exceptionnel de la figure royale féminine telle qu'elle entend paraitre, toujours à la limite des normes institutionnelles imposées par la couronne. C'est tout le problème de l'expression d'une gloire au féminin qui est alors posé et de la représentation d'une épouse royale qui, pour être au sommet de la hiérarchie sociale, n'en est pas moins une éternelle sujette ${ }^{1}$.

2 L'ambition de la commanditaire jointe à l'habileté de l'artiste donne naissance à une œuvre complexe dotée de multiples clés de lecture. L'une d'entre elles réside dans le message politique fort que la reine mère veut faire passer, à ses contemporains comme à la postérité, dans cet hymne à sa gloire. Pour mener une telle entreprise, il était nécessaire de penser la représentation du féminin et du masculin dans la sphère du pouvoir, penser l'éclat de la reine en ménageant la susceptibilité de Louis XIII, penser l'efficacité d'un cycle dont la compréhension immédiate ne devait pas atténuer le succès ultérieur. Les atouts de Marie de Médicis tiennent autant à la renommée du peintre qu'à la qualité de l'équipe dont elle s'entoure pour diriger l'opération : son principal conseiller Richelieu, son argentier l'abbé Maugis, ainsi que l'érudit Peiresc prennent une part active à l'aventure, comme en témoigne l'étroite correspondance qu'ils entretiennent avec Rubens pendant toute la durée des travaux. Ils ont en effet grand intérêt à voir leur protectrice retrouver du crédit auprès du roi, et sont très attentifs à l'élaboration et à la réalisation du programme pictural. Pour autant, si le cycle est à bien des égards une entreprise collective, la réussite en incombe essentiellement à la volonté de la commanditaire et au talent du peintre. À la tête de l'opération, la reine mère peut être 
créditée de son succès, de la même façon que les tableaux, pourtant travaillés en atelier, sont signés de Rubens.

Prévue pour être dévoilée à l'occasion du mariage d'Henriette de France avec le prince de Galles en mai 1625, l'œuvre est jusque-là gardée secrète " parce que Sa Majesté ne désire pas qu'on voye [les tableaux] que le tout ne soit faict et posé où ils doivent estre $»^{2}$. Le cycle, inauguré alors que tous les représentants des grandes cours européennes sont réunis pour assister aux noces royales, est ainsi pensé comme un tout. Prises indépendamment, les toiles ne font que relater des épisodes de la vie de Marie, mais reliées entre elles par un jeu complexe de correspondances, elles révèlent la substance du message délivré. Rendre compte des ambitions de la reine mère et analyser ce faisant le contenu politique de l'œuvre de Rubens requièrent donc de mettre en interaction composition picturale et structure du cycle.

4 Lorsque, en février 1622, Marie de Médicis commande à Rubens une série de tableaux destinés à orner l'aile occidentale du palais du Luxembourg, elle est à nouveau assurée d'une position de pouvoir à la cour. Après une régence qui lui permit de tenir, pendant plus de quatre ans (1610-1614), les rênes du gouvernement, après trois autres années où elle maintint son influence politique avec le titre de surintendante de l'État, après une longue période de brouille avec son fils qui, pour se débarrasser d'une mère trop présente, avait fait assassiner ses favoris Concini et Léonora Galigaï et l'avait exilée à Blois, après les deux guerres dites « de la mère et du fils » au cours desquelles les troupes royales affrontèrent celles de la reine mère, Marie de Médicis profite de la mort du connétable Albert de Luynes, en 1621, pour revenir en grâce auprès du jeune Louis XIII et s'installer à nouveau dans les arcanes du pouvoir, et au conseil royal. C'est dans ce processus de reconquête d'une place à laquelle elle n'a jamais renoncé que la reine demande à l'un des peintres les plus célèbres de son temps de composer une œuvre tout entière consacrée à sa gloire.

5 Le contexte est essentiel pour comprendre la portée politique d'une telle entreprise. Audelà du parcours biographique que retrace le cycle en célébrant les moments cruciaux de son existence, Marie de Médicis entend offrir au public un manifeste qui vise autant à justifier ses actes qu'à glorifier son être. Fidèle à la tradition médicéenne, la reine mère sait mobiliser les arts pour célébrer sa grandeur et servir sa politique. Commandé en 1615 à Salomon de Brosse, alors que rien ne semble faire obstacle à l'autorité de la douairière, le palais du Luxembourg est la première manifestation artistique de ce sentiment de puissance ${ }^{3}$. La décoration intérieure, dont la pièce maîtresse est constituée par le cycle de Rubens, en est le complément nécessaire. Outil de propagande, l'œuvre picturale, qui accompagne l'œuvre monumentale en voie d'achèvement lorsque la commande est passée à Rubens, s'avère autant un plaidoyer qu'un traité politique. Il s'agit de donner à la postérité un témoignage de sa grandeur et de transmettre à la cour et, au-delà, à toute l'Europe, un message politique de justification, de légitimation, mais aussi d'affirmation de son retour au pouvoir.

6 En visant ce double objectif, le cycle s'inscrit dans des temporalités différentes : comme plaidoyer, il est essentiellement conjoncturel, et s'adresse avant tout à ses contemporains, directement concernés par les affaires politiques en cours ; mais comme expression de la puissance d'une reine de France, il transcende le présent pour se projeter dans le futur, assurant à Marie de Médicis une gloire éternelle portée par la valeur artistique de sa représentation. Parfaitement conjoncturel et soigneusement structurel, ce cycle donne à voir Marie de Médicis telle qu'elle se pense, et veut être, en reine de 
France. Le sujet est ainsi replacé dans l'histoire, son histoire, qui est aussi celle du royaume de France, tout en étant étroitement lié au fonctionnement monarchique. Le portrait de celle qui fut princesse de Toscane, épouse d'Henri IV, mère de Louis XIII, régente du royaume et veuve éplorée d'un des plus puissants monarques de l'Europe est aussi une mise en image de la reine de France. À la dimension biographique s'ajoute donc une dimension institutionnelle qui complète et valorise la première. La part du récit est essentielle à la construction de l'œuvre car elle permet d'identifier l'héroïne du cycle et de personnaliser l'épopée. C'est bien Marie de Médicis qui mène la carrière d'une reine de France à son apogée; la puissance d'une telle position est le résultat d'un statut d'exception et d'une femme hors du commun. La narration de ses succès personnels permet à la commanditaire de magnifier son propre personnage, lequel tire cependant sa force de la dignité royale acquise dès ses noces, en 1600. La liberté prise avec la chronologie, comme la minutieuse reconstitution de scènes historiques, répondent à la nécessité de mettre l'accent sur les moments forts de la vie d'une princesse devenue souveraine.

7 Primordiale, l'histoire de Marie impose l'ordre de lecture du cycle, lequel commence avec la naissance de la princesse et s'achève alors que la réconciliation semble définitive entre la mère et le fils. Cette lecture, linéaire, de toiles qui se succèdent comme autant d'étapes ascensionnelles vers la gloire permet de rendre compte du cursus honorum de la fille du grand-duc François et d'insister sur le fait qu'au-delà du parcours d'une reine de France, c'est bien à Marie de Médicis qu'il convient de rendre hommage. Le souci d'exactitude historique, perceptible notamment dans les portraits de ceux qui participèrent aux événements ${ }^{4}$, renforce cette impression. Il est particulièrement manifeste dans deux des vingt-quatre toiles de la galerie. La Remise de l'anneau, qui représente le mariage par procuration, insiste sur le premier succès remporté dans cette marche au pouvoir (Fig. 13). Là, le peintre prend soin de donner de la scène une reconstitution extrêmement rigoureuse. Outre l'attention portée aux personnages présents lors de la cérémonie, et qui sont aisément identifiables dans la peinture, Rubens fait preuve d'un soin tout particulier pour le détail. La relation épistolaire suivie qu'il entretient avec son ami Peiresc, grand érudit proche du cercle de la reine et conseiller du peintre à maintes occasions, en porte témoignage. Donnant son avis sur le tableau dans une lettre datée du 27 octobre 1622, Peiresc écrit : « J'ai soumis à Mr l'abbé [Maugis] votre scrupule relativement à la présence de $\mathrm{Mr}$ le Grand, il m'a répondu que vous deviez suivre la vérité historique que vous connaissez bien $»^{5}$. En effet, Rubens, comme Peiresc d'ailleurs, se trouvait aux noces de Marie de Médicis à Florence. Les inquiétudes de l'artiste étant apaisées, Peiresc poursuit en attirant l'attention sur des points secondaires. Il conteste ainsi la présence du chapeau sur la tête du cardinal, qu'il propose de mettre sur un petit autel à côté, conformément au déroulement de la cérémonie, comme il conteste aussi le vêtement du prélat officiant : " Je me souviens très bien qu'il portait le pallium archiépiscopal sur la chasuble du prêtre » ${ }^{6}$. Aussi infimes que puissent paraître ces détails, Rubens en prend acte et modifie son œuvre : dans la peinture définitive, le chapeau a quitté la tête du cardinal, tandis que la tenue cérémonielle a été restituée dans sa forme originelle.

8 Ce souci d'authenticité se retrouve encore dans l'illustration d'une autre étape fondamentale du parcours royal: Le Couronnement, célébré en l'abbaye de Saint-Denis le 13 mai 1610, juste avant le départ programmé d'Henri IV pour la guerre et la remise de la régence à son épouse (Fig. 14). L'événement a été immortalisé dans une gravure célèbre de Léonard Gaultier à partir d'une composition de Nicolas Bollery, imprimée en $1610^{7}$. 
C'est de celle-ci que Rubens s'inspire, au point d'en offrir une reproduction assez fidèle, bien qu'inversée et en couleur ${ }^{8}$. Les personnages occupent une place identique, depuis les princesses du sang jusqu'à Henri IV, discrètement installé à un balcon, en retrait de la scène, et dont la présence/absence rappelle qu'au sacre d'une reine le roi, en principe, n'assiste pas, mais qu'il avait cette fois-ci voulu être là.

Aucune fantaisie n'intervient dans la présentation des personnages. De tels rituels sont à ce point codifiés que la place occupée, comme aussi la tenue vestimentaire, expriment la dignité. Les préséances doivent être respectées dès lors que l'artiste opte pour une reconstitution historique. C'est pourquoi tous les personnages du premier plan qui participent à la cérémonie peuvent être identifiés 9 . Dans les descriptions données de l'œuvre, la précision apportée à ce propos montre l'importance de l'enjeu. Cette toile est d'ailleurs la seule du cycle qui mobilise aussi explicitement le vocabulaire propre à la désignation des rangs. Ainsi par exemple, la notice de 1622 précise la qualité de celles qui portent la queue du manteau royal ${ }^{10}$, privilège réservé aux seules princesses du sang, et qui a fait l'objet de féroces disputes entre les dames de la cour ${ }^{11}$. À la fin du XVII siècle, Félibien souligne pour sa part le nombre de rangs de fleurs de lys qu'arborent les manteaux des princesses ${ }^{12}$, là encore manifestation de leur dignité à travers l'expression de leur appartenance à la famille de France; seules les filles de roi peuvent disposer de quatre rangs fleurdelisés. Dans un texte de 1704, l'attention est portée sur les couronnes, couronne fermée pour la reine Marguerite, fille de Henri II et première épouse de Henri IV, couronnes ducales pour les duchesses ${ }^{13}$. Il est significatif que les précisions apportées dans ces descriptions concernent justement les points sur lesquels se focalisent les querelles de préséances. Et le tableau de Rubens paraît si fidèle qu'il peut servir de preuve à l'occasion de tels conflits. Félibien, encore, rappelle dans sa notice que les princesses du sang réclamaient trois rangs de fleurs de lys sur leur manteau, " mais ne les purent obtenir $\aleph^{14}$, accordant ainsi au tableau une valeur démonstrative sur des questions qui restent encore très largement d'actualité près d'un siècle plus tard.

Pour autant, la minutieuse reconstitution de certaines scènes n'empêche pas quelques libertés prises avec la chronologie. Les deux opérations servent un même objectif: insister sur les moments forts de la vie de Marie, et articuler le récit biographique sur la qualité royale de la protagoniste. Si Marie doit son succès à sa valeur personnelle, si la grandeur de Marie tient à sa dignité royale, la structure même du cycle doit le mettre en exergue, en jouant, notamment, sur des temporalités différentes ${ }^{15}$. Ainsi, le temps est comprimé pour la longue période où Marie est princesse de Toscane, les vingt-sept premières années de sa vie tenant en deux toiles, La Naissance qui annonce un personnage d'exception et L'Éducation qui perfectionne des qualités innées. Il s'étire en revanche au moment du mariage : pas moins de quatre toiles traitent de cet épisode fondateur d'une autre vie, d'une nouvelle dignité. Aux trois étapes nécessaires pour sceller définitivement une alliance royale (fiançailles, mariage par procuration, célébration des noces en présence des époux, transformée ici en rencontre à Lyon afin que les compositions ne soient pas redondantes), Marie de Médicis en joint une quatrième - Le Débarquement à Marseille -, ajoutant ainsi une note personnelle à un parcours classique. Puis, à nouveau, le récit se fait elliptique. Un seul tableau résume les dix années passées aux côtés d'Henri IV : La Naissance du dauphin en 1601 ; tableau dans lequel la représentation des cinq autres enfants du couple royal, dans une corne d'abondance, symbolise cette décennie marquée par la maternité. La vie de reine régnante n'est ici qu'un tremplin pour atteindre la période la plus glorieuse, à savoir la régence. 
11 L'année 1610 marque un tournant décisif. La reine devient veuve, et régente du royaume. Les plus grandes compositions sont consacrées à ce moment capital qui voit la fin d'un roi et l'ascension d'une reine. La chronologie elle-même en est bouleversée : la régence est ici confiée à Marie avant qu'elle ne soit sacrée, l'intention du roi devenant action qui inaugure un règne. En effet, Henri IV tend à s'effacer de la scène politique après l'opération. Il n'est plus que spectateur lors du couronnement ; il quitte définitivement le monde dans le tableau suivant, cette composition paraissant d'ailleurs sanctionner un état de fait que sa présence discrète au couronnement annonçait déjà. Si, dans La Remise de la régence, le peintre concentre en réalité deux moments en un (volonté du roi de confier les rênes du gouvernement avant son départ pour la guerre et cession effective de l'autorité), l'ordre des toiles inscrit l'ascension de Marie dans un processus d'avènement. Comme pour le roi, Marie dispose du pouvoir avant d'être sacrée ${ }^{16}$.

12 Quatre toiles, donc, pour l'année $1610^{17}$, quatre toiles aussi pour relater les événements de la régence dans la réalisation définitive du cycle. Là encore, la chronologie n'est pas absolument rigoureuse: le double mariage espagnol, négocié et voulu par Marie de Médicis, est placé sous le gouvernement de la reine mère, entre 1610 et 1614, alors qu'il a lieu en 1615, un an après la majorité de Louis XIII et la fin officielle de la régence ${ }^{18}$. Procédant de la même façon que pour La Remise de la régence, l'intention devient réalité, la reine voulant faire de cet épisode historique son œuvre personnelle. Elle l'inscrit donc dans les années au cours desquelles elle exerce un pouvoir sans entrave. Il fut même question, dans un premier temps, d'accorder à l'événement plusieurs séquences, avant de réduire le programme à deux, puis une seule toile ${ }^{19}$. Ces tergiversations montrent l'importance que Marie de Médicis accordait à une entreprise qui engagea la politique européenne d'alors, et fut menée à bien malgré la forte opposition que le projet rencontra à la cour. Il n'y a pas d'année phare dans cette séquence, mais un grand nombre de toiles pour une très courte période. Le temps de la régence est, dans la vie d'une reine de France, un moment privilégié où elle met en œuvre tous les acquis de sa souveraineté en exerçant l'autorité. Cette situation d'exception pour une femme focalise l'attention sur huit scènes au total, en y comptant les quatre tableaux relatifs à l'année 1610. Par contre, après la majorité de Louis XIII qui met officiellement fin à son mandat, la reine semble se retirer des affaires. Aucune allusion n'est faite à l'influence qu'elle exerce encore dans le gouvernement avec le titre de surintendante de l'État pendant les trois années suivantes. La narration enchaîne directement sur les malheurs de Marie, veuve éplorée, fragile, victime. La reine régente, qui a elle-même succédé à la reine régnante, fait place à la reine douairière dont le seul lien effectif à la couronne sur le plan institutionnel tient dans le titre royal, qu'elle conserve, et dans son douaire assigné sur des terres du domaine. Tous les états d'une reine de France sont ainsi illustrés, et c'est cette fois en veuve, douairière, que Marie, dépouillée de toute autorité, se soumet aux coups du sort. Elle subit désormais les épreuves envoyées par le destin dans une attitude passive qui contraste fortement avec la gestuelle, volontaire, des années de pouvoir. Le statut de la reine continue de servir Marie en justifiant ses actes.

13 Si le récit biographique oriente la lecture du cycle chronologiquement, le portrait d'une reine de France permet de proposer un autre sens de visite, par un jeu de correspondance des toiles qui se répondent d'un côté à l'autre de la galerie. Cette lecture en zigzag, fondée sur le thème de la paix, met en évidence la mission pacificatrice traditionnellement assignée à l'épouse royale ${ }^{20}$. 

d'une reine de France, permettent d'envisager une division en croix de l'ensemble du cycle, dans le sens de la longueur d'une part, de la largeur d'autre part ${ }^{21}$. Le premier axe est longitudinal. Sur le mur de gauche en entrant se déroule l'existence de Marie en tant que femme, princesse de Toscane puis épouse d'Henri IV, tandis que la partie droite concerne sa vie de veuve. La toile centrale, placée au fond, en constitue le point de basculement, par les thèmes qu'elle traite comme par sa composition: à gauche le roi s'élève dans les Cieux pendant que la reine, à droite, devenue veuve, reçoit la régence des mains de la France. Le second axe, lui, est transversal. Les premiers tableaux en entrant représentent une femme hors du pouvoir, parce qu'elle n'est pas encore mariée ou parce qu'elle est douairière dépouillée de la régence ; ceux du fond, au contraire, traitent d'une femme placée au cœur du pouvoir en tant qu'épouse ou que régente. La rupture s'établit cette fois très précisément au milieu de la galerie par la représentation du mariage d'un côté, de la majorité de Louis XIII de l'autre, les deux portes d'entrée et de sortie de la scène politique. Marie de Médicis articule ainsi dans la conception du cycle données personnelles et données institutionnelles. Les unes comme les autres sont conditionnées par la nature féminine de la princesse, qui détermine son statut et donc sa marge d'action. Si la reine mère est capable d'en jouer, elle doit aussi surmonter un certain nombre de difficultés inhérentes à la place de la femme dans un monde de pouvoir réputé masculin.

Consacrer un cycle entier à la figure royale féminine pose des problèmes iconographiques qui sont loin d'être négligeables. Le premier tient à ce que la reine est, par la dignité qui est sienne, une incarnation de la monarchie. Elle ne détient pas le pouvoir souverain, mais elle en représente la grandeur. Elle peut donc en être l'expression sans qu'il y ait de confusion avec celui qui a hérité du trône. D'où, d'ailleurs, cette aptitude à remplacer un roi déficient. De fait, " si l'autorité leur manque, elles en ont au moins toute la représentation extérieure $»^{22}$. Pour autant, les reines brillent toujours d'une lumière empruntée, les rois « faisant luire sur elles les préeminences et les rayons qui eclatent de leurs Majestez en diverses manieres ${ }^{23}$. La qualité d'une reine ne se pense pas sans le roi qui l'a faite telle, et c'est bien l'existence de celui-ci qui donne à la princesse toute sa dimension. Prendre la reine pour seul sujet revient alors à reléguer à l'arrière-plan celui qui en constitue pourtant la caution. Une telle opération comporte toujours le risque d'une interprétation en termes d'usurpation, car elle réserve à l'épouse royale les signes extérieurs de la souveraineté. Il est cependant difficilement concevable de dresser le portrait d'une reine de France en faisant abstraction de sa dignité. Et Marie entend d'autant moins renoncer aux possibilités offertes par sa position royale qu'une souveraine, en France, semble n'avoir de place dans la mémoire nationale qu'à condition qu'elle ait gouverné en nom et place du roi, qu'elle joue donc, pour un temps, un rôle masculin. roi, tous deux expressions de la souveraineté, tous deux glorifiés par l'exercice du pouvoir. Ce constat rejoint un problème plus général, celui de la représentation des femmes dont la position traditionnelle - et en cela l'identifiant social - est affectée à l'espace domestique, mais dont l'élévation tient à leur aptitude à s'imposer dans un univers masculin. L'inverse n'est pas vrai : les hommes ne gagnent rien à montrer leur capacité à remplacer une épouse absente. La question est d'ailleurs posée en termes d'aptitude des femmes à exercer des fonctions masculines (définies par leur inscription 
dans l'espace public, comme par exemple des activités professionnelles) et non selon la capacité masculine à pallier la déficience féminine ${ }^{24}$. L'espace domestique, s'il n'est pas sans vertu, est assurément sans gloire.

17 Or c'est de sa gloire que Marie de Médicis veut laisser une image à la postérité. Plutôt qu'en épouse accomplie, elle entend se montrer en femme de pouvoir, comme l'exprime magistralement le contraste entre l'unique toile consacrée à son existence d'épouse et la forte proportion de scènes relatives à ses années de régence. Reine puissante par une position qui, à bien des égards, doit revenir au roi, la mère de Louis XIII joue subtilement de sa féminité. C'est parce qu'elle est femme que, veuve, elle devient impuissante ; c'est parce qu'elle est femme, aussi, que son destin est exceptionnel, hors du cadre imposé par un statut d'inférioritée 25 .

18 Épouse et mère, Marie s'inscrit dans la lignée de ces dames illustres qui ont marqué l'histoire. Son ambition est manifeste dans le choix des statues proposées pour orner le dôme surmontant le portail du palais du Luxembourg. Puisées dans l'Antiquité ou le passé glorieux de la France, « ce sont toutes des reines très célèbres, femmes ou mères de grands rois ${ }^{26}$, d'Olympias, mère d'Alexandre le Grand, à Blanche de Castille, mère de saint Louis. Leur royauté est un critère de sélection et celles qui n'eurent pas l'heur de posséder ce rang furent éliminées, telle $\mathrm{Mammoea}^{27}$ un moment envisagée mais finalement exclue parce qu' " elle ne fut pas une épouse, mais simplement une mère d'empereur $\aleph^{28}$. Dès l'entrée de l'édifice, en façade, Marie de Médicis souhaite donner le ton de ce que célèbre, par ailleurs, la présence même du bâtiment ${ }^{29}$. Ici, la gloire de la reine mère est étroitement associée aux deux premiers Bourbons, Henri IV et Louis XIII, ce qui n'est déjà plus le cas dans la galerie peinte par Rubens. Les références aux femmes célèbres se passent désormais de la puissance des monarques; les modèles sont choisis dans la mythologie afin d'inscrire Marie dans un cycle héroïque ${ }^{30}$.

La commanditaire joue ainsi sur tous les tableaux, de la reine, de la femme, de l'héroïne, pour occuper une place que l'institution n'accorde qu'au monarque. Si la souveraineté dont elle est une des incarnations renvoie implicitement au monde, masculin, du pouvoir, le thème de la paix tout aussi omniprésent est sans conteste un attribut féminin de la figure royale. Reine de paix et souveraine, Marie est selon les circonstances femme soumise ou dominatrice. Les vêtements qu'elle porte sont davantage qu'un effet de style : ils renvoient à sa position dans l'État. Habillée de couleurs chatoyantes ou de blanc lorsqu'elle est en mesure d'imposer sa volonté, et que ses actions ne reviennent qu'à son mérite, la reine est en veuve, toute vêtue de noir, lorsque le destin décide pour elle. Ainsi, l'entrée effective dans la régence, alors qu'Henri IV abandonne ce bas monde, est effectuée par une femme en deuil. Marie n'est en rien responsable de la situation, bien que ce soit par son mérite que le roi lui ait confié, à deux tableaux de là, la garde du royaume. Les rumeurs concernant sa participation à l'assassinat sont de la sorte étouffées, démenties par l'attitude d'humilité et d'impuissance qu'elle adopte. Pour autant, ces accusations ne sont pas prises à la légère, et Rubens doit modifier la composition de L'Apothéose d'Henri IV afin de ne pas prêter le flanc à la critique diffamatoire. Approuvé dans l'ensemble, le tableau suscite une réserve : «On y a trouvé d'autres difficultés que celle de l'intervention des Parques [...]. Elles y figureraient très bien s'il n'existait pas certains bruits sinistres qui se sont répandus dans ce temps contre la reine et qui n'étaient très certainement que des calomnies. Il vaudrait mieux cependant d'éviter une occasion de les rappeler $»^{31}$. 
20 En vêtement de veuve, encore, Marie remet la régence à son fils désormais majeur et se dépouille du pouvoir. Les scènes suivantes la représentent en noir, pour La Fuite de Blois, pour Le Traité d'Angoulême, pour La Conclusion de la paix. Toutes ces années de rébellion contre son fils se transforment ainsi en épreuves imposées à une femme démunie, et donc déchargée de toute responsabilité. L'habit de veuve escamote la reine et propose une explication - une justification, même - pour cette période si délicate à traiter qu'elle a fait l'objet des « sujets réservés » et non encore arrêtés lorsque la réalisation du cycle fut confiée à Rubens ${ }^{32}$.

21 À l'inverse, la reine est en blanc lorsqu'elle exerce le pouvoir, au temps de la régence. Elle quitte son costume de deuil sitôt le gouvernement en mains. La Prise de Juliers ou La Félicité de la régence montrent le triomphe d'une femme maîtresse de ses actes et responsable de ces actions d'éclat. C'est assurément à elle que le royaume doit une si talentueuse administration. Et c'est à nouveau en blanc que l'on retrouve Marie dans l'ultime scène consacrée à sa biographie, alors que La Réconciliation pleine et entière après la mort du connétable signe la fin de l'ostracisme et engage un processus de reconquête du pouvoir (Fig. 15). Le vêtement, en lui-même, manifeste son triomphe, tant sur les événements que sur la volonté du roi. Sortie sans dommage des épreuves, elle retrouve une place qu'elle n'aurait jamais dû quitter : celle du pouvoir.

Marie entend donc transmettre à la postérité l'image d'une femme forte que l'adversité a grandie, comme elle entend aussi confirmer à ses contemporains son retour sur la scène politique. Femme dont les qualités supérieures en font l'égale des rois, elle s'impose à la tête de l'État au-delà des réserves imposées à son sexe. L'ambiguïté de la représentation des reines en tant qu'incarnation de la souveraineté, le paradoxe d'une gloire féminine puisée dans des valeurs masculines et, finalement, le recours à la mythologie pour transformer l'histoire en épopée hérö̈que permettent les compositions les plus audacieuses. De la femme de pouvoir au souverain, le pas n'est pas explicitement franchi. Mais la substitution est très largement suggérée.

23 À l'entrée de la galerie, au-dessus de la porte, Marie de Médicis, représentée à l'antique, casque en tête et sceptre en main, est encadrée à droite par le portrait de son père, le grand-duc François Ier, et à gauche par celui de sa mère l'archiduchesse Jeanne d'Autriche (Fig. 16). Ces trois compositions donnent le ton de l'œuvre en articulant représentations historiques et figures mythologiques; la reine mère s'apparente ici aux dieux de l'olympe tandis que le grand-duc et son épouse appartiennent à leur siècle. En plaçant d'emblée Marie de Médicis dans un registre héroïque, le peintre entend signifier que le portrait de la reine est ici mythifié, et qu'il ne faut voir dans le cycle qu'une allégorie de la puissance royale. Cette perspective est renforcée par la scène des trois Parques filant le destin de Marie, qui introduit le récit biographique. Il semble pourtant que le recours à la mythologie serve un projet plus ambitieux. Là où les générations postérieures voient une Marie de Médicis en Pallas ${ }^{33}$, en Bellone ${ }^{34}$, ou en Minerve ${ }^{35}$, se profile en réalité une reine triomphante. La Description des tableaux de la galerie donnée en 1622 dévoile les intentions de la reine mère: «La roine sera peinte comme une reine triomphante l'armet en teste, le sceptre en main, dessoubz ses pieds des armes, casques, corselets, masses d'armes, tambours, au dessus de sa teste deux amours avecq des ailles de papillon, marque d'immortalité tenant une couronne de laurier sur la teste de la Royne pour monstrer que sa Gloire est immortelle et dedans le Ciel deux renommées tenans des triomphes publians ses vertus et la gloire de la bonne conduite du gouvernement de l'Estat, au dessoubz de ses pieds sera escript HIC EST ILLA disant voicy la plus grande 
royne de la terre, la plus rare vertu du monde qui n'a point eu de pareilles en tous les siecles de la posterité ${ }^{36}$. Plus que l'image elle-même, la notice qui s'y rapporte installe résolument Marie en lieu et place du roi. Le champ lexical du pouvoir mobilisé à travers les termes de triomphe, vertu, gloire, gouvernement, autant que les attributs retenus (armet, sceptre, couronne...) dressent le portrait d'un monarque célébré par les armes et le gouvernement. La description, de fait, conviendrait parfaitement à une image du roi. Mieux, elle paraîtrait plus adaptée ; plus classique peut-être, mais aussi plus crédible. Au vocable « reine » peut aisément se substituer celui de « roi » sans que le texte perde de sa cohérence. C'est bien là l'enjeu du cycle voulu par Marie : être représentée de telle sorte qu'elle puisse, finalement, trôner en souveraine.

Cette manifestation de puissance ne fait pas de la reine un personnage asexué : le sein droit dénudé que comporte la peinture rappelle la féminité de celle qui, par son mérite, accède au plus haut niveau de l'état, et évite que l'identification au monarque soit trop directe. Le décalage entre l'image et son commentaire, tous deux parfaitement contemporains, révèle les intentions : faire de Marie à la fois une femme et une reine de pouvoir sans qu'on puisse l'accuser d'outrepasser ses droits. L'accumulation de thèmes offre une solution en proposant pour chaque tableau, et pour le cycle dans son ensemble, plusieurs clés de lecture qui détournent, accessoirement, du sens profond que recèle l'œuvre. Car Marie n'hésite pas à prendre la place du roi, encore, dans l'ordre des scènes qui marquent 1610 et annoncent son avènement, ou dans le choix des actes qui caractérisent la régence. Le Triomphe de Juliers et L'Échange des princesses appartiennent résolument au registre des relations extérieures, domaine traditionnellement réservé au monarque dans la répartition des attributions au sein du couple royal. Si la guerre ou la négociation d'alliances sont inhérentes à tout gouvernement et reviennent donc à la régence, le fait que Marie insiste sur cet aspect de son action politique n'est pas anodin. La mission théoriquement assignée à ceux qui exercent le pouvoir par intérim est avant tout de transmettre au nouveau roi un royaume tel que son prédécesseur l'a laissé. Les actions d'éclat sont l'apanage des rois. La reine trouve pourtant là l'occasion de marquer son passage. Elle s'impose en chef de guerre dans Le Triomphe de Juliers, en majesté dans la Félicité de la régence, tableau si complexe que Rubens lui-même le considère sans danger ${ }^{37}$. La composition n'est cependant pas dénuée d'ambiguïté : Marie, en manteau royal, trône sous un dais, en tenant un sceptre d'une main et une balance de l'autre, symboles de la justice et de l'exercice de l'autorité souveraine. Au XVIIIe siècle, le public ne s'y trompe pas; il s'agit $d^{\prime}$ » un tableau représentant la reine dans son lit de justice $»^{38}$. Le message est passé à la postérité.

Femme d'autorité au point d'occuper la place du roi, Marie se veut légitime dans le conseil royal qu'elle rejoint en 1622, au moment de la commande passée au peintre flamand. Derrière l'apologie perce donc un discours visant à cautionner son retour au pouvoir. L'œuvre de Rubens participe ainsi d'une vaste opération qui mobilise également les lettres, afin de justifier l'attitude de la reine mère pendant les années noires de 1617-162039. Mais elle va plus loin en proclamant le triomphe de sa commanditaire. Le cycle, construit de façon circulaire, est présenté comme un éternel recommencement. Les Parques qui filent le destin de l'héroïne, en prélude à l'épopée, prennent soin de ne pas couper le fil, contrairement à la tradition : l'histoire relatée ici n'a pas de fin. Et au mouvement descendant de cette première scène répond l'envolée de la dernière image : dans les sphères célestes, la reine mère inaugure un nouveau règne, éternel. 
La construction en boucle tient aussi à la relation, conçue en phases alternatives, que la protagoniste entretient avec le pouvoir. Princesse impuissante, elle devient souveraine; douairière sans autorité, elle s'impose en reine mère. Les différents statuts qu'une épouse royale est susceptible de connaître (princesse, régnante, régente, douairière) en font une reine accomplie, justifiant qu'elle occupe, durablement, une place au plus haut niveau. L'effacement de Marie, sortie du gouvernement par la majorité de Louis XIII, ne constitue ainsi qu'une phase transitoire qui préfigure une nouvelle ascension. La Réconciliation pleine et entière, ultime scène narrative du cycle, consacre la victoire définitive de la reine (Fig. 15). Construite sur le modèle d'une assomption de la Vierge ${ }^{39}$, où la mère est accueillie par le Fils, la scène suggère une renaissance, après la Dormition. Le vêtement blanc de la reine/Vierge remplace le costume de deuil lié à une période douloureuse, et l'assistance retrouve une femme de pouvoir comme aux jours glorieux de la régence. Les démons de la discorde et de la calomnie sont définitivement chassés, effaçant du même coup le souvenir funeste de ces dernières années. Légitime aux côtés du roi, la reine triomphe, tant des épreuves traversées que des difficultés qu'une femme peut rencontrer pour s'imposer à la tête de l'État. Ses vertus personnelles, sa dignité royale, son parcours et sa qualité de mère soulignée par le groupe que forment au coin supérieur gauche du tableau une jeune femme et ses enfants, justifient amplement non seulement la réconciliation, mais au-delà un rôle dans le gouvernement. S'il est un personnage tout désigné pour prodiguer des conseils à son fils, c'est bien la reine mère; ce que reconnaît finalement Louis XIII, rendu à la raison par la disparition du connétable et l'attitude soumise de Marie aux heures noires. Il l'accueille dans la sphère, supérieure, de la souveraineté, et la consacre une seconde fois.

Le cycle se conclut sur une autre référence à l'iconographie religieuse : le couronnement de la Vierge. Répétition, allégorique cette fois, de la scène qui ouvrit à la reine mère la voie du pouvoir, cette ultime image proclame la toute-puissance de celle qui se veut - et se retrouve - au même niveau qu'un roi. Elle partage la couronne, comme elle partage le trône. La gloire céleste est éternelle ; le triomphe de Marie n'est pas seulement total, il est définitif.

\section{BIBLIOGRAPHIE}

Le cycle de Marie de Médicis par Rubens.

Structure générale du cycle. Les axes de lecture

ANDIA Béatrice de, 1991, «L'art, fer de lance des rois », dans : Marie-Noëlle Baudouin-Matuszek (dir.), Marie de Médicis et le palais du Luxembourg, Paris, Délégation à l'action artistique de la ville de Paris, pp. 11-25.

BAILLY Jacques, 1751, Catalogue des tableaux du cabinet du Roy au Luxembourg, Paris, Prault père (première édition 1750).

BAUDOIN-MATUSZEK Marie-Noëlle (dir.), 1991, Marie de Médicis et le palais du Luxembourg, Paris, Délégation à l'action artistique de la ville de Paris. 
CARMONA Michel, 1988, Marie de Médicis, Paris, Fayard.

COSANDEY Fanny, 2000, La Reine de France, symbole et pouvoir, Paris, Gallimard.

- 2003, « Les femmes en monarchie : épouses ou héritières ? ", Le Genre face aux mutations, Actes du colloque tenu à l'Université de Rennes II du 26 au 28 novembre 2002, PUR, pp. 201-209.

Description de la galerie du Palais du Luxembourg, 1704, Paris, Jean Boudot (signé MDM, ce texte est attribué à Moreau de Mautour par Jacques Thuillier, 1969).

DUCHESNE André, 1609, Les Antiquités et recherches de la grandeur et de la majesté des Roys de France, Paris.

FELIBIEN André, 1705, Entretiens sur les vies et sur les ouvrages des plus excellents peintres, Londres, David Mortier (première édition Paris, 1666-1688).

FOUCART Jacques et THUILLIER Jacques, 1969, Rubens. La galerie Médicis au palais du Luxembourg, Paris, Robert Laffont.

GODINEAU Dominique, 2003, Les Femmes dans la société française (XVIe-XVIIIe siècles), Paris, Armand Colin.

Henri IV et la reconstruction du royaume, 1989, Paris, RMN-Archives Nationales

MAMONE Sara, 1990, Paris et Florence, deux capitales du spectacle pour une reine, Marie de Médicis, Paris, Le Seuil.

ROOSES Max et RUELENS Ch., 1897-1909, Correspondance de Rubens et documents épistolaires concernant sa vie et ses œuvres, Anvers, J. Buschmann.

THUILLIER Jacques, 1969, " La 'Galerie Médicis' de Rubens et sa genèse : un document inédit », La Revue de l'Art, n4, p. 52-62.

VON SIMSON Otto Georg, 1968, Il ciclo di Maria de Medici, Milan, Fratelli Fabri.

WASCHEK Mathias, 1995, « Comment la providence divine guida Marie de Médicis : tentative de réinterprétation du cycle Rubens dans son contexte spatial », Les Yeux du corps, Paris, CIPS, pp. 71-88.

\section{NOTES}

1. L'analyse proposée ici est le prolongement d'une étude du cycle menée dans le cadre d'un travail sur la reine de France. Nombre de conclusions sont donc reprises sans être développées. Pour une perspective d'ensemble de l'œuvre dans son contexte institutionnel, voir Cosandey 2000.

2. Lettre de Richelieu à Maugis, mai 1623, dans Rooses et Ruelens 1897-1909, t. III : 163.

3. Voir Mamone 1990; Béatrice de Andia, " L'art, fer de lance des rois ", dans BaudouinMatuszek (dir), 1991 : 17.

4. Toutes les descriptions du cycle, contemporaines ou postérieures à sa réalisation, sont attentives à l'identité des personnages qui s'y rencontrent.

5. Rooses et Ruelens 1897-1909, t. III : 60. Lettre en italien, traduction française de Rooses et Ruelens [texte italien : « Io ho fatto intendere al Sr Abbate il scrupulo di V.S. Intorno la presenza de Mr Le Grand ; ma egli dice que V.S. deve seguire la verita de l'historia che le sara nota »].

6. Rooses et Ruelens 1897-1909, t. III : 60. Lettre en Italien, traduction française de Rooses et Ruelens [texte italien: "Ben mi ricordo che gli haveva il pallio archiepiscopale sopra la casula sacerdotale »]. 
7. La gravure a pour titre : Le sacre et couronnement de Marie de Médicis. Cf. Henri IV et la reconstruction du royaume $1989: 407$

8. Le rapprochement a été opéré par Otto Georg von Simson 1968 : 72.

9. Et ceci d'autant plus que les relations de la cérémonie désignent nominalement la place occupée. Voir par exemple Les cérémonies et ordre tenu au sacre et couronnement de la Royne Marie de Médicis..., s.l., 1610.

10. Thuillier 1969 : 58. Le compte rendu est à ce point précis que Jacques Thuillier y voit là une série d'indications pour réaliser la toile, non encore peinte à la date du texte.

11. Voir la querelle relative au mariage de Marie-Thérèse en 1660, BnF, Ms Fr. 20826, fol. 27.

12. Félibien 1705 (1ère édition 1666-88) : 323.

13. Description de la Galerie du palais du Luxembourg 1704.

14. Félibien 1705 (1ère édition 1666-88) : 323.

15. L'analyse du cycle narratif ne prend pas en compte les cinq petits tableaux placés à l'entrée de la galerie, et qui encadrent les scènes proprement biographiques, sans intervenir directement dans le récit chronologique.

16. Le principe d'instantanéité de la succession fait qu'en France un règne commence au moment précis où un roi disparait. Le couronnement est donc toujours postérieur et n'est, sur le plan juridique, qu'une confirmation de ce que donne le droit de naissance.

17. À condition de considérer que le Conseil des dieux est rattaché à l'année 1610. Ce tableau occupe cependant une position ambiguë. Il peut s'interpréter comme l'annonce d'un bon gouvernement (Description de la Galerie du palais du Luxembourg 1704 ; Bailly 1751), mais il peut aussi figurer " le Concert des dieux pour les mariages réciproques de la France et d'Espagne " (Description de 1622), sens que retient finalement Jacques Thuillier (Thuillier 1969: 60).

18. Seule la signature du contrat de mariage, le 22 août 1612, se déroule pendant la régence.

19. Foucart et Thuillier 1969 ; Rooses et Ruelens 1897-1909, t. III : 9-10 ; lettre de Maugis à Peiresc du 1er août 1622.

20. Waschek 1995 ; Cosandey 2000.

21. Voir schéma en annexe (p. 83). L'entrée de la salle est en haut du schéma.

22. De l'autorité des Reynes de France, Bnf, Ms Fr. 14013, fol. 14.

23. Duchesne 1609 : 566 .

24. L'historiographie récente se fait l'écho de cette problématique. Voir à ce sujet la précieuse synthèse proposée par D. Godineau qui tend à montrer que les femmes exercent, bien plus qu'on ne le croyait, des fonctions masculines (Godineau 2003).

25. S'il convient de relativiser cette infériorité, la reine partageant avec le roi une position dominante dans l'ordre social, la femme est cependant toujours placée hiérarchiquement audessous de son époux. En cela, elle est l'égale et l'inférieure de l'homme. Cf. Cosandey 2003.

26. Lettre de Peiresc à Rubens du 26 mai 1622, Rooses et Ruelens 1897-1909, t. II : 419. Lettre en italien, traduction française de Rooses et Ruelens [texte italien : « Tutte regine, molto illustri, moglie et madri di principi grandi »].

27. Appelée aussi Mammée, elle est la mère d'Alexandre Sévère.

28. Lettre du 9 juin 1622, Rooses et Ruelens 1897-1909, t. II : 437. Lettre en italien, traduction française de Rooses et Ruelens [texte italien : « che non fu moglie d'imperatore anzi solamente madre con tutto ch'avesse la qualita di Augusta »].

29. Les huit statues que devait réaliser le sculpteur Guillaume Berthelot n'étaient cependant toujours pas posées en 1631. Baudouin-Matuszek $1991: 193$.

30. C'est d'ailleurs cette lecture du cycle que Jacques Foucart et Jacques Thuillier privilégient dans leurs analyses. Foucart et Thuillier 1969.

31. Lettre de Peiresc à Rubens du 26 mai 1622. L'artiste, prenant acte de ces observations, remplace les Parques par Bellone, Rooses et Ruelens 1897-1909, t. II : 418. Lettre en italien, traduction française de Rooses et Ruelens [texte italien : " Altra difficolta non vi si e trovato, se non 
l'intervento delle Parche nel nuovo quadro ottavo, lequali vi starebbono benissimo senza certi rumori sinistri che si sparsero in que tempi contra la Regina, ben che calomnie certissime; ma sara meglio di vietarne l'occasione »].

32. Foucart et Thuillier 1969.

33. Félibien 1705 (1ère édition 1666-1688).

34. Description de la Galerie du palais du Luxembourg 1704.

35. Bailly 1751 (1750).

36. Ce tableau est considéré comme le premier du cycle. Description des tableaux de la Gallerie du palais de la Royne mere du Roy aux faubourgs St Germain les Paris, BnF, Ms Baluze 323, f॰ 54, publié par Thuillier 1969.

37. «Ce sujet, qui ne touche pas spécialement à la raison d'État de ce royaume, ne s'applique individuellement à personne; il a beaucoup plu, et je crois que si l'on s'était fié entièrement à moi, les autres sujets auraient mieux passé sans scandale ni murmure », Lettre de Rubens à Peiresc du 13 mai 1625, Rooses et Ruelens 1897-1909, t. III : 357. Lettre en italien, traduction française de Rosses et Ruelens [texte italien: Questo soggietto che non tocca la raggion di stato particolar di questo regno ne s'applica ad alcun individuo, ha piacciuto molto et io credo que se si fossero fidati intieramente di noi, che le cose toccante gli altri soccietti sarebbe passate meglio senza alcun scandalo o murmuratione].

38. Mémoires relatifs à la restauration des tableaux du Luxembourg consécutive à la nomination, le 18 septembre 1753, d'une commission chargée de superviser les opérations. Cf. Foucart et Thuillier $1969: 144$.

39. Sur la composition du cycle marial, cf. Cosandey, $2000: 341-346$.

\section{RÉSUMÉS}

En commandant à Rubens un cycle exclusivement consacré à sa gloire, Marie de Médicis entend affirmer à toute l'Europe son retour au pouvoir. Au-delà du récit biographique, l'œuvre picturale délivre un message politique fort qui justifie, et légitime, la reine mère dans le gouvernement. Pour parvenir à ses fins, et donner d'elle-même l'image d'une souveraine toute puissante sans transgresser ouvertement les principes monarchiques, Marie de Médicis joue de toutes ses qualités, de femme, de reine, de mère, dans un cycle complexe qui articule le registre narratif sur le registre institutionnel, et mêle figures allégoriques et compositions historiques. En ce début de $\mathrm{XVII}^{\mathrm{e}}$ siècle, c'est bien le problème de la représentation d'une femme de pouvoir qui est alors posé.

When she commissioned Rubens to paint an entire cycle paying homage to her, Maria de' Medici meant to inform all Europe of her return to power. Beyond the biographical narration, this work delivers a strong political message that justifies and legitimates her governmental role as QueenMother. For that purpose and to present herself as an omnipotent sovereign without overtly transgressing monarchical principles, she makes use of all her attributes. woman, queen, and mother - in this complex cycle of pictures connecting narrative to institutional elements and mixing allegorical figures with historical compositions. At the beginning of the $17^{\text {th }}$ century, this cycle thus raises the problem of the representation of a woman wielding power. 
INDEX

Mots-clés : Reine de France, Rubens, représentation monarchique, femmes de pouvoir, stratégie picturale

Index géographique : Palais du Luxembourg

\section{AUTEUR}

\section{FANNY COSANDEY}

Fanny COSANDEY est docteur de l'EHESS. Elle a fait sa thèse sur la reine de France $\left(\mathrm{XV}^{\mathrm{e}}-\mathrm{XVII}^{\mathrm{e}}\right.$ siècles). Dans le prolongement de cette étude, elle travaille actuellement selon deux axes : le rôle des femmes dans la construction politique à l'époque moderne ; les querelles de préséances à la cour de France, entendues comme la manifestation des tensions provoquées par l'émergence d'une modernité politique. Ces deux orientations s'inscrivent par ailleurs dans une perspective plus large concernant les relations entre patrimoine privé et puissance publique, entre famille royale, système dynastique et État monarchique. Elle a publié récemment : La Reine de France, symbole et pouvoir (Gallimard, 2000); Monarchies française et espagnole, XVI ${ }^{\mathrm{e}}-\mathrm{XVII} \mathrm{e}^{\mathrm{e}}$ siècles (avec Isabelle Poutrin; Atlande, 2001) ; L'Absolutisme en France. Histoire et historiographie (avec Robert Descimon ; Le Seuil, 2002). 